\title{
Naturalness of the relaxion mechanism
}

\author{
Andrew Fowlie, ${ }^{a}$ Csaba Balazs, ${ }^{a}$ Graham White, ${ }^{a}$ Luca Marzola ${ }^{b, c}$ and Martti Raidal ${ }^{b, c}$ \\ ${ }^{a}$ ARC Centre of Excellence for Particle Physics, \\ School of Physics and Astronomy, Monash University, \\ Wellington Road, Melbourne, Victoria 3800, Australia \\ ${ }^{b}$ National Institute of Chemical Physics and Biophysics, \\ Rävala 10, Tallinn 10143, Estonia \\ ${ }^{c}$ Institute of Physics, University of Tartu, \\ Ravila 14c, Tartu 50411, Estonia \\ E-mail: andrew.fowlie@monash.edu, csaba.balazs@monash.edu, \\ graham.white@monash.edu, luca.marzola@ut.ee, martti.raidal@cern.ch
}

ABSTRACT: The relaxion mechanism is a novel solution to the hierarchy problem. In this first statistical analysis of the relaxion mechanism, we quantify the relative plausibility of a QCD and a non-QCD relaxion model versus the Standard Model with Bayesian statistics, which includes an automatic penalty for fine-tuning. We find that in light of the hierarchy between the weak and Planck scales, relaxion models are favoured by colossal Bayes-factors. Constraints upon e.g., the vacuum energy during relaxation, however, shrink the Bayesfactors such that relaxion models are only slightly favoured. Including the bounds on $\left|\theta_{\mathrm{QCD}}\right|$ shatters the plausibility of the QCD relaxion model as it typically yields $\left|\theta_{\mathrm{QCD}}\right| \gg 0$. Finally, we augment our models with scalar-field inflation and consider measurements of inflationary observables from BICEP/Planck. We find that, all told, the Standard Model is favoured by huge Bayes-factors as the relaxion models require fine-tuning such that the Hubble parameter is less than the height of the periodic barriers. Thus, whilst we confirm that relaxion models could solve the hierarchy problem, we find that their unconventional cosmology is at odds with their plausibility.

Keywords: Beyond Standard Model, Higgs Physics

ArXiv EPrint: 1602.03889 


\section{Contents}

1 Introduction 1

2 Bayesian fine-tuning $\quad 3$

3 Analysis of relaxion potential $\quad 4$

$3.1\left|\theta_{\mathrm{QCD}}\right|$ in relaxion models $\quad 6$

$\begin{array}{lll}3.2 & \text { Finite-temperature effects } & 7\end{array}$

$\begin{array}{lll}3.3 & \text { Baryon asymmetry } & 7\end{array}$

4 Description of models $\quad 8$

4.1 The Standard Model with scalar-field inflation 8

4.1.1 Calculation of observables 9

$\begin{array}{llr}4.2 & \text { Relaxion models } & 9\end{array}$

$\begin{array}{lll}\text { 4.2.1 Relaxion physicality conditions } & 10\end{array}$

$\begin{array}{ll}\text { 4.2.2 Calculation of (electroweak and QCD) observables } & 11\end{array}$

5 Bayesian analysis $\quad 11$

$\begin{array}{lll}5.1 & \text { Evidences } & 14\end{array}$

$\begin{array}{lll}5.2 & \text { Observables } & 15\end{array}$

$\begin{array}{llr}6 & \text { Discussion and conclusions } & 17\end{array}$

\section{Introduction}

Graham et al. [1] recently proposed a relaxation mechanism [2-4] that solves the hierarchy problem [5-8] by utilising the dynamics of an axion-like field, dubbed the relaxion. In the Standard Model (SM), the hierarchy problem originates from quadratic corrections to the weak scale. Whereas supersymmetry cancels them with new quadratic corrections involving supersymmetric particles [9], the relaxion mechanism cancels them with the vacuum expectation value (VEV) of a relaxion field.

The ingenuity of the relaxion mechanism is that the dynamics of the relaxion field ensure a precise cancellation without patent fine-tuning of parameters or initial conditions. Within the relaxion paradigm, interactions between a complex Higgs doublet, $h$, and an axion-like field, $\phi$, govern the weak scale via the scalar potential [1]

$$
V=\left(\mu^{2}-\kappa\langle a\rangle \phi\right) h^{2}-m_{b}^{3}\langle h\rangle \cos \left(\frac{\phi}{f}\right)-m^{2}\langle a\rangle \phi+\lambda h^{4},
$$

where, because of quadratic corrections, we expect that the masses should be close to the cut-off $\Lambda$, i.e., $\mu^{2} \sim m^{2} \sim \Lambda^{2}, m_{b}$ and $f$ are coupling constants of dimension mass, $\langle a\rangle$ is 
the VEV of a spurion field that breaks a shift symmetry $\phi \rightarrow \phi+2 \pi f, \kappa$ is a dimensionless coupling, and $\langle h\rangle$ is the VEV of the Higgs field, which is a function of the relaxion field $\phi$.

Let us label the co-efficient of $h^{2}$ in the relaxion potential

$$
m_{h}^{2}(\phi) \equiv \mu^{2}-\kappa\langle a\rangle \phi,
$$

for convenience, such that the Higgs VEV may be written

$$
\langle h\rangle= \begin{cases}\sqrt{\frac{-m_{h}^{2}(\phi)}{2 \lambda}} & m_{h}^{2}(\phi)<0, \\ 0 & \text { otherwise. }\end{cases}
$$

If the Higgs VEV is non-zero, the cosine term provides a periodic barrier for the relaxion field with barriers separated by $2 \pi f$. In the unbroken phase in which $\langle h\rangle=0$, the barrier is down and the relaxion field slowly rolls down a linear potential. Once $m_{h}^{2}(\phi)<0$, however, the potential is such that the Higgs field acquires a VEV,$\langle h\rangle \neq 0$, breaking electroweak symmetry (EWSB) and raising the periodic barrier. The now-raised periodic barrier traps the relaxion field in a minimum. If the relaxion field cannot roll past a local minimum, it results in a weak scale of about

$$
\langle h\rangle \gtrsim f \frac{m^{2}\langle a\rangle}{m_{b}^{3}} .
$$

Thus this mechanism could result in $\langle h\rangle \ll M_{\mathrm{P}}$.

We require, inter alia, that the relaxion field dissipates energy as it rolls or else it would have sufficient kinetic energy to surmount the periodic barriers. In the relaxion paradigm, this is ensured by Hubble friction - a term analogous to a friction term in the EulerLagrange equation for the relaxion field originating from the expansion of the Universe see e.g. ref. [10]:

$$
\ddot{\phi}+3 H \dot{\phi}+\frac{\partial V}{\partial \phi}=0
$$

where $H$ is the Hubble parameter. If Hubble friction is substantial, the relaxion field could be in a slow-roll regime in which the acceleration $\ddot{\phi}$ can be neglected.

Ostensibly, the relaxion mechanism ameliorates fine-tuning associated with the weak scale, but Raidal et al. [11] stress that it could require a fine-tuned inflationary sector if the relaxion is the QCD axion because of constraints upon the Hubble parameter during inflation. Unfortunately, there is no consensus in high-energy physics on the appropriate measure of fine-tuning or about the logical foundations of fine-tuning arguments, despite their prominence. In earlier work to judge fine-tuning in relaxion models, Jaeckel et al. [12] developed a new formalism based on their intuition, whereas Raidal et al. [11] utilised common Barbieri-Giudice style measures [13, 14]. In section 2, we critique Jaeckel's approach and instead advocate a Bayesian methodology, discussed numerous times over the last decade in the context of fine-tuning in supersymmetric models [15-22]. In this methodology, in light of experimental data about the weak scale and inflation, we update our belief in a model with a Bayesian evidence. We further analyse the relaxion potential in section 3 . We describe our models - minimal relaxion models and the SM augmented by scalar-field inflation - in section 4 and calculate their Bayesian evidences in section 5. This is the 
first statistical analysis of a relaxion model. We close in section 6 with a brief discussion of our findings.

\section{Bayesian fine-tuning}

Bayesian statistics provides a logical framework for updating beliefs in scientific theories in light of data see e.g. ref. [23-25]. This methodology is becoming increasingly common in high-energy physics see e.g. ref. [26-69] and cosmology see e.g. ref. [70-72], and arguably captures the essence of the hierarchy problem [15-22] and the principle of Occam's razor see e.g. ref. [73]. We briefly recapitulate the essential details.

The Bayesian framework enables one to assign numerical measures to degrees of belief. To assess two models, $M_{a}$ and $M_{b}$, one begins by quantifying one's relative degree of belief in the models, prior to considering any experimental data. This is known as the prior odds,

$$
\text { Prior odds } \equiv \frac{P\left(M_{a}\right)}{P\left(M_{b}\right)},
$$

where $P(M)$ is one's prior belief in a model $M$. From the prior odds, we can calculate the posterior odds - one's relative degree of belief in the models updated with experimental data,

$$
\text { Posterior odds } \equiv \frac{P\left(M_{a} \mid D\right)}{P\left(M_{b} \mid D\right)},
$$

where $D$ represents experimental data e.g., in this work data from BICEP/Planck. The prior odds and the posterior odds are related by a so-called Bayes-factor:

$$
\text { Posterior odds }=\text { Bayes-factor } \times \text { Prior odds }
$$

By applying Bayes' theorem, it can be readily shown that the Bayes-factor is a ratio of probability densities,

$$
\text { Bayes-factor } \equiv \frac{p\left(D \mid M_{a}\right)}{p\left(D \mid M_{b}\right)},
$$

where the probability densities in question are known as Bayesian evidences or just evidences. The evidence for a model $M$ can be calculated by Bayes' theorem and marginalisation,

$$
\mathcal{Z} \equiv p(D \mid M)=\int p(D \mid M, \boldsymbol{p}) \cdot p(\boldsymbol{p} \mid M) \prod \mathrm{d} \boldsymbol{p}
$$

where $\boldsymbol{p}$ are the model's parameters, $p(D \mid M, \boldsymbol{p})$ is a so-called likelihood function - the probability density of our observed data given parameters $\boldsymbol{p}$ - and $p(\boldsymbol{p} \mid M)$ is our prior density for the model's parameters $\boldsymbol{p}$.

The likelihood function is uncontroversial as its form is dictated by the nature of an experiment and it is a critical ingredient in Bayesian and frequentist statistics. The role and form of the prior density, however, remain contentious issues. In as much as it is possible, we pick objective priors that reflect our knowledge or ignorance about a parameter and respect rational constraints from e.g., symmetries. 
We calculate Bayes-factors for the SM augmented with scalar-field inflation $(\mathrm{SM}+\sigma)$ versus relaxion models. The final step - that of updating one's prior odds with a Bayesfactor to find one's posterior odds — is left to the reader. That is not to say that a Bayes-factor is independent of any prior choices - it is in fact a functional of the priors for the parameters of the models in question.

Before closing, we briefly discuss attempts to quantify fine-tuning in a relaxion model by Jaeckel et al. [12] and by Raidal et al. [11]. Raidal et al. employed Barbieri-Giudice style measures of fine-tuning $[13,14]$. Whilst intuitive, such measures lack a logical foundation, though emerge in intermediate steps in a calculation of the Bayesian evidence [15-22]. Jaeckel et al. developed a novel measure of electroweak fine-tuning, $F$, based on the fraction of a model's parameter space, $\boldsymbol{p}$, that predicts a weak scale less than that observed:

$$
\frac{1}{F} \equiv \frac{V_{v(\boldsymbol{p}) \leq v}}{V}=\frac{\int \theta(v-v(\boldsymbol{p})) \prod \mathrm{d} \boldsymbol{p}}{\int \prod \mathrm{d} \boldsymbol{p}} .
$$

This measure contrasts with Barbieri-Giudice measures in that it considers a model's entire parameter space rather than a single point in it. Jaeckel's measure, however, depends on one's choice of parameterisation or measure for the parameter space. ${ }^{1}$

Curiously, Jaeckel's measure in eq. (2.6) is reminiscent of the Bayesian evidence if one considers measurements of the weak scale, especially if one writes (unnecessary) normalisation factors for the priors,

$$
\mathcal{Z}=\frac{\int p(v \mid M, \boldsymbol{p}) \cdot p(\boldsymbol{p} \mid M) \prod \mathrm{d} \boldsymbol{p}}{\int p(\boldsymbol{p} \mid M) \prod \mathrm{d} \boldsymbol{p}} \quad \text { vs. } \quad \frac{1}{F}=\frac{\int \theta(v-v(\boldsymbol{p})) \prod \mathrm{d} \boldsymbol{p}}{\int \prod \mathrm{d} \boldsymbol{p}} .
$$

The differences are that Jaeckel et al. pick a step-function for the likelihood for the weak scale, $v$, and omit a measure for the volume of parameter space, i.e., a prior. In other words, by following their noses and attempting to formulate fine-tuning in a logical manner, Jaeckel et al. create an ersatz Bayesian evidence.

\section{Analysis of relaxion potential}

Let us further analyse the relaxion potential in eq. (1.1),

$$
V=\left(\mu^{2}-\kappa\langle a\rangle \phi\right) h^{2}-m_{b}^{3}\langle h\rangle \cos \left(\frac{\phi}{f}\right)-m^{2}\langle a\rangle \phi+\lambda h^{4} .
$$

As in ref. [74], for simplicity we consider only linear terms in the relaxion field $\phi$. The equations $\partial V / \partial \phi=0$ and $\partial V / \partial h=0$ result in a transcendental equation,

$$
\sin (\phi / f)=\frac{f \kappa\langle a\rangle}{m_{b}^{3}}\left(\frac{m^{2} / \kappa+\langle h\rangle^{2}}{\langle h\rangle}\right) .
$$

By graphing as in figure 1, one finds that if there is a solution, it lies in the interval $\langle h\rangle_{\min } \leq\langle h\rangle \leq\langle h\rangle_{\max }$ where

$$
\langle h\rangle_{\min }=\frac{m_{b}^{3}-\sqrt{m_{b}^{6}-4 \kappa m^{2}\langle a\rangle^{2} f^{2}}}{2 \kappa\langle a\rangle f} \text { and }\langle h\rangle_{\max }=\sqrt{\frac{-\mu^{2}+\kappa\langle a\rangle\langle\phi\rangle_{\max }}{2 \lambda}},
$$

\footnotetext{
${ }^{1}$ However, Jaeckel et al. [12] includes a general discussion of parameterisation in the context of finetuning.
} 


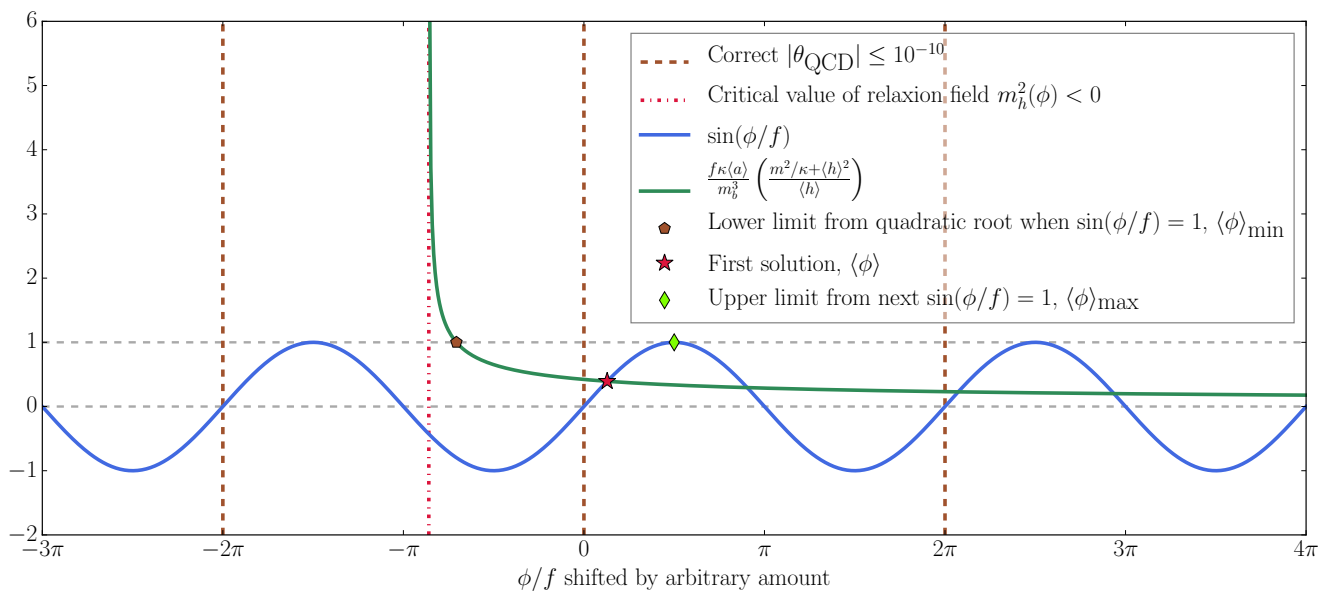

Figure 1. Graphing the left-hand side (blue line) and right-hand side (green line) of the transcendental equation in eq. (3.1). The solution (red star) lies in the interval in eq. (3.2), marked by a brown pentagon and a green diamond. In unusual cases, the second point at which the right-hand side equals plus one (not shown) may be a sharper bound. The $\phi / f$-axis is shifted such that correct $\left|\theta_{\mathrm{QCD}}\right|$ occurs at small multiples of $2 \pi$ (vertical brown dashed lines) close to the solution. EWSB is broken once the critical value of the relaxion field is surpassed (vertical red dot-dashed line).

and

$$
\langle\phi\rangle_{\min }=\frac{2 \lambda\langle h\rangle_{\min }^{2}+\mu^{2}}{\kappa\langle a\rangle} \text { and }\langle\phi\rangle_{\max }=(2 n+1 / 2) \pi f
$$

where $n$ is the smallest integer such that $\left.\langle\phi\rangle_{\max }\right\rangle\langle\phi\rangle_{\min }$. If the square-root is imaginary, there are no solutions, otherwise, there are zero to four solutions inside the interval, which must be identified numerically. The interval results from recognising that a solution must lie between the point at which the right-hand side of eq. (3.1) equals plus one, matching the maximum of the left-hand side, and the subsequent point at which the latter is again maximal. If required, one can improve this interval with piece-wise expressions by graphing. In some cases, the positive quadratic root, similar to that for $\langle h\rangle_{\min }$, is a sharper bound for $\langle h\rangle_{\max }$. If the barrier height is substantial, the lower bound reduces to the approximation for $\langle h\rangle$ in eq. (1.2), that is,

$$
\langle h\rangle_{\min } \approx f \frac{m^{2}\langle a\rangle}{m_{b}^{3}} \quad \text { if } \quad \frac{4 \kappa m^{2}\langle a\rangle^{2} f^{2}}{m_{b}^{6}} \ll 1 .
$$

This implies that $\kappa\langle a\rangle \ll m_{b}^{3} /(4 f\langle h\rangle)$. A necessary (though not sufficient) condition for solutions to the transcendental equation is that the root in eq. (3.2) must be real,

$$
\frac{4 \kappa m^{2}\langle a\rangle^{2} f^{2}}{m_{b}^{6}} \leq 1
$$

In other words, the relaxion mechanism ensures that the weak scale is independent of quadratic corrections to the Higgs mass from a cut-off or unknown high-scale physics, 
solving the hierarchy problem. In fact, the Higgs VEV is bounded by an expression that is independent of the Higgs mass,

$$
\langle h\rangle_{\min } \leq\langle h\rangle \leq\langle h\rangle_{\max } \leq \sqrt{\langle h\rangle_{\min }^{2}+\kappa\langle a\rangle f \pi / \lambda} .
$$

The Higgs mass $\mu^{2}$ and any corrections affect the position of $\langle h\rangle$ inside this interval, but not the interval itself. The width of this interval is typically small such that numerically solving for the VEV of the Higgs field inside the interval may be unnecessary.

Unfortunately, if the relaxion is the QCD axion, we expect that barrier height $m_{b}^{3}$ is connected to QCD, such that $m_{b} \sim \Lambda_{\mathrm{QCD}}$, resulting in

$$
\langle a\rangle \lesssim\langle h\rangle \times 10^{-18} \simeq 10^{-16} \mathrm{GeV},
$$

where we impose an experimental limit on the QCD decay constant, $f \gtrsim 10^{9} \mathrm{GeV}$, and pick $m \simeq 1 \mathrm{TeV}$ in eq. (3.4). Thus achieving a small weak scale requires a tiny spurion VEV,$\langle a\rangle \lll M_{\mathrm{P}}$. Such a small coupling may be natural as it breaks a shift-symmetry see e.g. ref. [75]; however, there may be issues due to the gauge symmetry at the basis of the construction [76, 77].

\section{$3.1\left|\theta_{\mathrm{QCD}}\right|$ in relaxion models}

Let us investigate whether a relaxion model might resolve the strong-CP problem [78] by explaining $\left|\theta_{\mathrm{QCD}}\right| \lesssim 10^{-10}[79]$. Prima facie, the expression for $\left|\theta_{\mathrm{QCD}}\right|$ is simple see e.g. ref. [80],

$$
\left|\theta_{\mathrm{QCD}}\right|=\mid\langle\phi\rangle / f \quad \text { on }-\pi \text { to } \pi \mid .
$$

Numerically, however, this cannot be used for calculating $\left|\theta_{\mathrm{QCD}}\right|-$ as $\langle\phi\rangle / f$ is substantial, there is a breakdown in numerical precision in expressions such as $\langle\phi\rangle / f \bmod 2 \pi$. Instead, we find the principal solution for $\left|\theta_{\mathrm{QCD}}\right|$,

$$
\left|\theta_{\mathrm{QCD}}\right|=\arcsin \left|\frac{f \kappa\langle a\rangle}{m_{b}^{3}}\left(\frac{m^{2} / \kappa+\langle h\rangle^{2}}{\langle h\rangle}\right)\right|,
$$

by utilising eq. (3.1).

The minimum $\left|\theta_{\mathrm{QCD}}\right|$ obtainable occurs at the minimum of the right-hand side of eq. (3.1), such that, if there is a solution at that point, $\sin (\phi / f)$ is as close to zero as possible. Thus we find that

$$
\min \left|\theta_{\mathrm{QCD}}\right|= \begin{cases}\arcsin \left|\frac{2\langle a\rangle f m \sqrt{\kappa}}{m_{b}^{3}}\right| & \text { if }\langle h\rangle_{\text {minima }} \leq\langle h\rangle_{\max }, \\ \arcsin \left|1-\frac{\kappa\langle a\rangle f \pi}{2 \lambda\langle h\rangle_{\min }^{2}}+\cdots\right|=\pi / 2-\sqrt{2 \epsilon}+\cdots & \text { otherwise }\end{cases}
$$

where $\langle h\rangle_{\text {minima }}=m / \sqrt{\kappa}$ which minimises the right-hand side of eq. (3.1) and the second line is never less than the first line. The terms represented by the ellipses are higher powers of $\eta$ and $\epsilon$,

$$
\langle h\rangle_{\max } \approx\langle h\rangle_{\min }(1+\epsilon) \quad \text { where } \quad \epsilon \equiv \frac{\kappa\langle a\rangle f \pi}{2 \lambda\langle h\rangle_{\min }^{2}} \ll 1 \quad \text { and } \quad \eta \equiv \frac{\kappa\langle h\rangle_{\min }^{2}}{m^{2}} \ll 1 .
$$


We expand to first order in $\epsilon$ and neglect all powers of $\eta$. Eq. (3.10) originates from considering that the minimum possible $\left|\theta_{\mathrm{QCD}}\right|$ would occur at the minimum of the righthand side of eq. (3.1) if that minima occurred at $\phi / f \approx 2 n \pi$ and not $\phi / f \approx(2 n+1) \pi$, such that $\left|\theta_{\mathrm{QCD}}\right| \approx 0$ and not $\left|\theta_{\mathrm{QCD}}\right| \approx \pi$. If that minima occurs, however, outside the interval for the possible solutions for $\langle h\rangle$, it is impossible. In that case, the right-hand side of eq. (3.1) is monotonic inside the interval for the possible solutions for $\langle h\rangle$ and we consider the right-hand side evaluated at $\langle h\rangle_{\max }$ from eq. (3.6). As the general expression is rather complicated, we apply the approximations in eq. (3.11), which are reasonable for phenomenologically viable points. In fact, phenomenologically viable points are always in the second regime in which $\langle h\rangle_{\text {minima }}=m / \sqrt{\kappa} \gg\langle h\rangle_{\max }$, such that $\min \left|\theta_{\mathrm{QCD}}\right| \approx \arcsin 1=$ $\pi / 2$. This is confirmed in our numerical analysis.

\subsection{Finite-temperature effects}

In this paper and in the literature so far, the relaxion mechanism was analysed at zero temperature. ${ }^{2}$ Finite-temperature effects could, however, non-trivially affect the relaxion potential in eq. (1.1):

- Non-perturbative effects responsible for the induced effective potential of the relaxion are temperature dependent [82]. However, since this affects only the heights of the barriers and not their spacing and since Hubble friction during inflation is typically substantial, it is unclear whether finite-temperature effects would impact the viability of the relaxion mechanism.

- Finite-temperature corrections to the effective potential would alter the shape of the potential (increasing the gradients of the slopes), possibly delaying the onset of EWSB [83]. If EWSB is delayed until a late time (corresponding to a lower temperature after reheating), it could constrain when inflation must start through the requirement that it lasts at least $50 e$-folds after EWSB. Furthermore, the flat regions of the zero-temperature inflaton potential are strongly modified by finitetemperature effects.

- We find that the reheating temperature in our relaxion models is typically of order $10^{10} \mathrm{GeV}$. At such a high temperature, electroweak symmetry could be easily restored, with the effect of further hindering the viability of the model.

Clearly all the mentioned finite-temperature effects have the potential to impose further constraints on the relaxion model parameter space, to an extent that will be quantified in following projects.

\subsection{Baryon asymmetry}

We observe a significant baryon asymmetry in our Universe. Sakharov [84] demonstrated that generating this asymmetry — baryogenesis - would require a departure from thermal

\footnotetext{
${ }^{2}$ We note, however, that ref. [81] considers finite-temperature effects in an alternative relaxion mechanism.
} 
equilibrium, $\mathcal{C}$ and $\mathcal{C P}$ violation, and baryon number violation. In the relaxion paradigm, however, the final 50 or so $e$-folds of inflation occur during or immediately after EWSB and inevitably wash-out any potential net baryon number generated in this process see e.g. ref. [85]. Novel mechanisms that invoke multi-step phase transitions are also ruled out since the fields must be in the final SM vacuum at the end of inflation. ${ }^{3}$ Scenarios in which the inflaton itself could generate the required baryon asymmetry see e.g. ref. [86, 87] also appear to be incompatible with the relaxion mechanism because of the further constraints implied by the already heavily constrained dynamics of the inflaton. Finally weak sphalerons are also exponentially VEV suppressed [88] after the electroweak phase transition which means that any subsequent baryogenesis scenario would have to rely on a different source of baryon and lepton number violation. As we shall see, these difficulties would strengthen our conclusions about the viability of inflation in the present framework.

\section{Description of models}

We apply Bayesian model comparison to three models: the SM augmented with single-field scalar-field inflation $(\mathrm{SM}+\sigma)$, a QCD relaxion model and a general relaxion model. For other relaxion models, see e.g., ref. [74, 89-96]. Ultimately, we wish to find whether the relaxion mechanism ensures that a relaxion model is favoured by the Bayesian evidence versus the SM. In each model, all scalar-fields receive quadratic corrections to their masses from a cut-off, $\Lambda$, which lies close to the Planck scale.

\subsection{The Standard Model with scalar-field inflation}

The SM Higgs sector is described by two bare Lagrangian parameters $-\mu^{2}$ and $\lambda-$ in the SM Higgs potential,

$$
V_{h}=\mu^{2}|h|^{2}+\lambda|h|^{4}
$$

and a cut-off at which the bare parameters are specified, $\Lambda$. We augment the SM with mixed inflation, a canonical model of scalar-field inflation see e.g. ref. [97]. Mixed inflation is described by an inflaton mass, $m_{\sigma}^{2}$, and quartic coupling, $\lambda_{\sigma}$, in a potential

$$
V_{\sigma}=\frac{1}{2} m_{\sigma}^{2} \sigma^{2}+\lambda_{\sigma} \sigma^{4}
$$

and the number of $e$-folds, $N_{e \text {-fold }}$. We denote this model by $\mathrm{SM}+\sigma$.

Note that in the $\mathrm{SM}+\sigma$ model, the evidence approximately factorises into a factor for the weak scale and a factor for the inflationary observables, $r, n_{s}$ and $A_{s}$,

$$
\begin{aligned}
\mathcal{Z} & =p\left(M_{Z}, r, n_{s}, A_{s} \mid \mathrm{SM}+\sigma\right) \\
& \approx p\left(M_{Z} \mid \mathrm{SM}+\sigma\right) \cdot p\left(r, n_{s}, A_{s} \mid \mathrm{SM}+\sigma\right) \\
& =p\left(M_{Z} \mid \mathrm{SM}\right) \cdot p\left(r, n_{s}, A_{s} \mid \sigma\right)
\end{aligned}
$$

as the measurements are independent and model parameters that affect inflationary observables do not affect the weak scale and vice-versa, with the exception of the cut-off, $\Lambda$, which results in quadratic corrections to the inflaton mass and the Higgs mass.

\footnotetext{
${ }^{3}$ One could in principle have a multi-step phase transition that departed from the SM vacuum for baryogenesis and later returned to it but this somewhat undermines the motivation for relaxion models.
} 


\subsubsection{Calculation of observables}

For comparison with measurements from Planck in our statistical analysis, we calculate inflationary observables via the so-called slow-roll parameters [98]:

$$
\epsilon(\sigma) \equiv \frac{M_{\mathrm{P}}^{2}}{16 \pi}\left(\frac{V^{\prime}(\sigma)}{V(\sigma)}\right)^{2} \quad \text { and } \quad \eta(\sigma) \equiv \frac{M_{\mathrm{P}}^{2}}{8 \pi} \frac{V^{\prime \prime}(\sigma)}{V(\sigma)}
$$

where a prime indicates a derivative with respect to the inflaton field $\sigma$. Inflation finishes once the inflaton field reaches a value $\sigma_{f}$ such that $\epsilon\left(\sigma_{f}\right)=1$. The number of $e$-folds desired before inflation ends (and in the case of the relaxion, after EWSB),

$$
N_{e-\text { fold }} \simeq \frac{-8 \pi}{M_{\mathrm{P}}^{2}} \int_{\sigma_{i}}^{\sigma_{f}} \frac{V(\sigma)}{V^{\prime}(\sigma)} \mathrm{d} \sigma
$$

determines the inflaton field at the beginning of inflation, $\sigma_{i}$. The number of $e$-folds desired should be $N_{e \text {-fold }} \gtrsim 50$. The spectral index, $n_{s}$, and the ratio of scalar to tensor perturbations, $r$, may be written to first order in the slow-roll parameters as see e.g. ref. [99]

$$
n_{s}=1-6 \epsilon\left(\sigma_{i}\right)+2 \eta\left(\sigma_{i}\right) \text { and } \quad r=4 \pi \epsilon\left(\sigma_{i}\right) .
$$

The normalisation of the potential governs the amplitude of scalar perturbations and the Hubble parameter,

$$
\begin{aligned}
A_{s} & =\frac{1}{M_{\mathrm{P}}^{6}} \frac{128 \pi}{3} \frac{V\left(\sigma_{i}\right)^{3}}{\mid V^{\prime}\left(\sigma_{i}\right)^{2}}, \\
H & =\sqrt{\frac{V\left(\sigma_{i}\right)}{3 M_{\mathrm{P}}^{2}}},
\end{aligned}
$$

but cannot affect $r$ or $n_{s}$. The normalisation of the scalar perturbations is arbitrary and varies in the literature. For comparison with Planck data, we pick that of the Planck experiment see e.g. ref. [100]. We include a quadratic correction to the inflaton mass to include a dominant quantum contribution to fine-tuning - but otherwise our formulas are tree-level. We solve for the inflaton field at the beginning and end of inflation, $\sigma_{i}$ and $\sigma_{f}$, with numerical methods.

The mass of the $Z$ boson - which represents the weak scale - is calculated in the usual manner,

$$
M_{Z}^{2}=\frac{-g^{2}}{2 \lambda}\left(\mu^{2}+\beta \Lambda^{2}\right)
$$

where $\beta$ is a loop factor. The QCD phase is an input parameter.

\subsection{Relaxion models}

We consider two relaxion models described by the potential in eq. (1.1). In the first model, we do not identify the relaxion with the Peccei-Quinn axion that solves the strong CP problem [78], whereas in the second model, the relaxion is indeed the Peccei-Quinn axion. 
For a necessary epoch of low-scale inflation after the relaxion mechanism, we extend the relaxion potential in eq. (1.1) by the most general renormalisable single-field inflaton potential see e.g. ref. [11] with an inflaton field $\sigma$,

$$
V=m_{3}^{3} \sigma+\frac{1}{2} m_{2}^{2} \sigma^{2}+\frac{1}{3} m_{1} \sigma^{3}+\frac{1}{4} \lambda_{\sigma} \sigma^{4} .
$$

We suppose that pre-inflation multi-field dynamics dictate that inflation begins at the origin, $\sigma=0$, as in Raidal et al. [11]. This introduces only four parameters: four couplings in the potential - the desired number of $e$-folds, $N_{e \text {-fold }}$, is not an input parameter. We, furthermore, tune a dressed vacuum energy, $\rho$, such that the cosmological constant vanishes in the vacuum, i.e., $V(\langle\sigma\rangle, \ldots)+\rho=0$. Thus, low-scale inflation with $H \lll M_{\mathrm{P}}$ is achieved provided $V(\sigma=0)=\rho \lll M_{\mathrm{P}}^{4}$. This implies that the potential must be fine-tuned such that $|V(\langle\sigma\rangle)| \lll M_{\mathrm{P}}^{4}$.

The cosmological constant poses an infamous fine-tuning problem see e.g. ref. [101]. In almost all known models, agreement with measurements of the cosmological constant requires extreme fine-tuning between a bare cosmological constant in the Lagrangian, $\rho_{0}$, quantum corrections and contributions from spontaneous symmetry breaking i.e., $V(\langle\sigma\rangle, \ldots)$. Because all models that we consider suffer from this fine-tuning problem, fine-tuning penalties from the cosmological constant would approximately cancel in ratios of Bayesian evidences. We ensure that the second epoch of inflation cannot spoil the relaxion mechanism by applying conditions on the Hubble parameter during inflation.

\subsubsection{Relaxion physicality conditions}

There are parameter points for which the back-reaction to EWSB fails to trap the relaxion field in a minimum. If that were the case, the relaxion mechanism would fail and the point would be in severe disagreement with observations. Graham et al. list conditions required for a successful relaxion mechanism [1]:

$$
\begin{array}{rlr}
H^{2} M_{\mathrm{P}}^{2} & >\frac{\mu^{2} m^{2}}{\kappa} & \text { (vacuum energy) } \\
H & <m_{b} & \text { (barriers form) } \\
H^{3} & <m^{2}\langle a\rangle & \text { (classical beats quantum) }
\end{array}
$$

We assign zero likelihood to a point that violates the resulting condition,

$$
\sqrt{\frac{\mu^{2} m^{2}}{\kappa}}<M_{\mathrm{P}} \min \left(m_{b}, m^{2 / 3}\langle a\rangle^{1 / 3}\right)
$$

Graham et al. also list the conditions

$$
\begin{array}{rrr}
N_{e \text {-fold }} & \gtrsim \frac{H^{2}}{\kappa\langle a\rangle^{2}} & \text { (inflation lasts long enough) } \\
\kappa\langle a\rangle \mu^{2} f & \sim m_{b}^{3}\langle h\rangle & \text { (barrier heights) }
\end{array}
$$

We assume that a first epoch of inflation is provided by the slow-rolling relaxion fields, and cosmological constant later cancelled when the Higgs and relaxion fields acquire VEVs, and 
that this epoch provides an acceptable Hubble parameter, as described in ref. [11]. The latter condition is unnecessary as we solve the potential with numerical methods, checking whether a solution exists. To avoid destroying the periodic barriers, the second epoch of inflation must, however, satisfy,

$$
H<m_{b}
$$

where, as before, $m_{b}$ is related to the height of the periodic barriers. The barriers result from a phase-transition to a QCD condensate (or a condensate associated with similar nonQCD dynamics). A Hubble parameter $H>m_{b}$ would reverse that transition, destroying the barriers see e.g. ref. [1].

\subsubsection{Calculation of (electroweak and QCD) observables}

We calculated the VEVs of the Higgs and relaxion fields with numerical methods based on bisecting the interval in eq. (3.2), from which we calculated the mass of the $Z$-boson,

$$
M_{Z}=g\langle h\rangle
$$

and $\left|\theta_{\mathrm{QCD}}\right|$ (see section 3.1). In the non-QCD relaxion model, $\left|\theta_{\mathrm{QCD}}\right|$ is an input parameter. The calculations for the inflationary observables were identical to those in the $\mathrm{SM}+\sigma$ model.

\section{$5 \quad$ Bayesian analysis}

We calculated Bayesian evidences for our $\mathrm{SM}+\sigma$ model and relaxion models with (Py)MultiNest [102-105], which utilises the nested sampling algorithm [106, 107] for MonteCarlo integration in Bayesian evidences in eq. (2.5) (though delta-functions were first integrated by hand). ${ }^{4}$ This requires two ingredients: a likelihood function and a set of priors. Our likelihood function, summarised in table 1, was a product of at most five factors:

- weak-scale: a likelihood function for measurements of the mass of the $Z$-boson [79]. In the SM, this is approximated by a delta-function and integrated by hand. In a relaxion model, this is impossible, as there is no analytic expression for the $Z$-boson mass as a function of the Lagrangian parameters.

- conditions: if a relaxion model (i.e., a point in a relaxion model's parameter space) violates physicality conditions in section 4.2.1, we assign a likelihood of zero, since it would be in stark disagreement with observations.

- decay: a likelihood function for the experimental lower-limit on $f_{a}$, the axion decay constant, approximated by a step-function see e.g. ref. [108].

- theta: a likelihood function for the experimental upper-limit on $\left|\theta_{\mathrm{QCD}}\right|$, approximated by a step-function [79].

\footnotetext{
${ }^{4}$ We utilised importance sampling, picked 1000 live points and a stopping criteria of 0.01 in MultiNest.
} 


\begin{tabular}{|ccc|}
\hline Parameter & Measurement & Likelihood function \\
\hline weak-scale & & \\
\hline$M_{Z}$ & $91.1876 \pm 0.0021 \mathrm{GeV}[79]$ & Dirac in SM, Gaussian in relaxion \\
\hline decay & & \\
\hline$f_{a}$ & $f_{a} \gtrsim 10^{9} \mathrm{GeV}[108]$ & Step-function \\
\hline theta & & \\
\hline$\left|\theta_{\mathrm{QCD}}\right|$ & $\left|\theta_{\mathrm{QCD}}\right| \lesssim 10^{-10}[79]$ & Step-function \\
\hline inflation & & Step-function \\
\hline$r$ & $r<0.12$ at $95 \%[109]$ & Gaussian \\
$n_{s}$ & $0.9645 \pm 0.0049[110]$ & Gaussian \\
$\ln \left(10^{10} A_{s}\right)$ & $3.094 \pm 0.034[110]$ & \\
\hline
\end{tabular}

Table 1. Likelihoods included in our Bayesian evidences for the scale of electroweak symmetry breaking, the axion decay constant, $\left|\theta_{\mathrm{QCD}}\right|$ and BICEP/Planck measurements of inflationary observables. Note that we neglect statistical correlations in Planck measurements of inflationary observables.

- inflation: a likelihood for the spectral index, $n_{s}$, the ratio of scalar to tensor perturbations, $r$, and the amplitude of scalar perturbations, $A_{s}$, from Planck and BICEP measurements [109, 110]. For simplicity, we neglect correlations amongst Planck measurements and impose an upper-limit for the scalar-to-tensor ratio.

We applied the likelihoods incrementally in five calculations per model: only weak-scale; adding conditions; adding a lower-bound on the axion decay constant, decay; adding an upper bound on $\left|\theta_{\mathrm{QCD}}\right|$, theta; and finally adding BICEP/Planck measurements in inflation. This enabled us to assess the individual impacts of the constraints.

We picked uninformative scale-invariant priors for the dimensionful Lagrangian parameters and cut-off because we are ignorant of their scale, a linear prior for $\left|\theta_{\mathrm{QCD}}\right|$, reflecting a shift-symmetry, and a linear prior for $N_{e \text {-fold }}$ because the number of $e$-folds is already a logarithmic quantity. Our prior ranges are summarised in table 2. All massive parameters $-\mu^{2}$ and $m^{2}$ and inflaton masses - receive quadratic corrections from a cut-off, such that we expect that without fine-tuning $\mu^{2} \sim m^{2} \sim \Lambda^{2}$. The main difference between the priors for our QCD relaxion model and general relaxion model is that in the former, the barrier height is related to the QCD scale, whilst in the latter, it is no greater than about the weak scale. Furthermore, we assigned zero prior weight to corners of parameter space in which a relaxion Lagrangian parameter with dimension mass exceeded the cut-off scale, $\Lambda$, as in an effective field theory such corners may be considered inconsistent or implausible. This eliminated approximately $18 \%$ of the prior volume in table 2 .

By picking scale-invariant priors for the Lagrangian parameters, we implicitly acknowledge our lack of information about a possible UV theory that might generate them. If there were sufficient reason to believe that a particular mechanism might generate them, imprint- 


\begin{tabular}{|c|c|c|}
\hline Parameter & Prior & \\
\hline \multicolumn{3}{|l|}{$\mathrm{SM}+\sigma$} \\
\hline$\mu^{2}$ & $\log$ & $10^{-40}, 1$ \\
\hline$\lambda$ & $\log$ & $10^{-4}, 4 \pi$ \\
\hline$\Lambda^{2}$ & $\log$ & $10^{-4}, 1$ \\
\hline$m_{\sigma}^{2}$ & $\log$ & $10^{-40}, 1$ \\
\hline$\lambda_{\sigma}$ & $\log$ & $10^{-20}, 4 \pi$ \\
\hline$N_{e \text {-fold }}$ & Linear & 50,500 \\
\hline$\left|\theta_{\mathrm{QCD}}\right|$ & Linear & $0, \pi$ \\
\hline \multicolumn{3}{|l|}{ QCD relaxion } \\
\hline$\mu^{2}$ & $\log$ & $10^{-40}, 1$ \\
\hline$\lambda$ & $\log$ & $10^{-4}, 4 \pi$ \\
\hline$\Lambda^{2}$ & $\log$ & $10^{-4}, 1$ \\
\hline$\kappa$ & $\log$ & $10^{-4}, 4 \pi$ \\
\hline$\langle a\rangle$ & $\log$ & $10^{-50}, 1$ \\
\hline$m_{b}$ & $\log$ & $10^{-1} \Lambda_{\mathrm{QCD}}, 10 \Lambda_{\mathrm{QCD}}$ \\
\hline$m^{2}$ & $\log$ & $10^{-40}, 1$ \\
\hline$f$ & $\log$ & $10^{-50}, 1$ \\
\hline$m_{1}$ & $\log$ & $10^{-100}, 1$ \\
\hline$m_{2}^{2}$ & $\log$ & $10^{-100}, 1$ \\
\hline$m_{3}^{3}$ & $\log$ & $10^{-100}, 1$ \\
\hline$\lambda_{\sigma}$ & $\log$ & $10^{-40}, 4 \pi$ \\
\hline \multicolumn{3}{|c|}{ Non-QCD relaxion, as for QCD relaxion except } \\
\hline$m_{b}$ & $\log$ & $10^{-6}\langle h\rangle, 10^{-1}\langle h\rangle$ \\
\hline$\left|\theta_{\mathrm{QCD}}\right|$ & Linear & $0, \pi$ \\
\hline
\end{tabular}

Table 2. Priors for parameters in SM augmented with scalar-field inflation $(\mathrm{SM}+\sigma)$ and relaxion models. Masses are in Planck units. Furthermore, regions in parameter space in which a dimensionful relaxion Lagrangian parameter exceeded a cut-off were assigned zero prior weight.

ing distinguishing correlations between parameters, it should be reflected in one's choice of priors. In supersymmetric models, for instance, the knowledge of particular breaking mechanisms such as minimal supergravity (mSUGRA) results in relations between Lagrangian parameters at the GUT scale that must be acknowledged in the choice of suitable priors. Likewise, the identification of a UV-completion of the relaxion model could, in principle, ameliorate the fine-tuning of the scenario as the Bayes-factor is a functional of the priors, however, to-date we know of no compelling reason to pick anything other than scale-invariant priors. 


\section{$5.1 \quad$ Evidences}

The evidences and Bayes-factors for the $\mathrm{SM}+\sigma$ and relaxion models are summarised in table 3. We find that, considering only a measurement of the weak scale (i.e., weak-scale), relaxion models are favoured by colossal Bayes-factors of about $10^{30}$. This is similar to findings for the constrained minimal supersymmetric SM versus the SM [17], and was expected, as the SM with a Planck-scale cut-off makes an egregious generic prediction for the weak scale.

The physicality conditions (conditions in section 4.2.1) dramatically impact the preference for relaxion models. The conditions wipe-out a fraction of the relaxion models' parameter spaces and shrink the Bayes-factors by about $10^{-28}$. The preference for relaxion models versus the SM almost entirely disappears. In other words, despite their success in solving the hierarchy problem, relaxion models are hamstrung by severe fine-tuning associated with their physicality conditions.

The preference for the QCD relaxion model is further damaged by measurements of the axion decay constant, decay, and the $\left|\theta_{\mathrm{QCD}}\right|$, theta. The latter results in approximately zero preference for the QCD relaxion model as it predicts that $\left|\theta_{\mathrm{QCD}}\right| \approx \pi / 2$ (see eq. (3.10)). The preference of about $10^{2}$ for a non-QCD relaxion model versus the SM is unaffected by decay and theta.

The final data-set of inflationary observables (inflation) also impairs the credibility of the considered relaxion models. Low-scale inflation, required in the relaxion paradigm, suffers severe fine-tuning as it requires a light scalar, and thus results in partial Bayesfactors of about $10^{-30}$ for relaxion models versus the SM. Thus, all data considered, the $\mathrm{SM}+\sigma$ model is favoured by a Bayes-factor of at least about $10^{25}$. We note that eq. (4.14) results in an approximate limit of $\mu^{2} \sim m^{2} \lesssim\left(10^{8} \mathrm{GeV}\right)^{2}$, such that by chance

$$
\frac{\mu^{2}}{\beta M_{\mathrm{P}}^{2}} \frac{m^{2}}{\beta M_{\mathrm{P}}^{2}} \sim \frac{M_{Z}^{2}}{\beta M_{\mathrm{P}}^{2}}
$$

The factors are in fact approximately the fractions of parameter space in which a scalar mass is fine-tuned to be so light versus a cut-off, $M_{\mathrm{P}}$. Thus tuning two scalar masses $-\mu^{2}$ and $m^{2}$ - in a relaxion model to be $\mu^{2} \sim m^{2} \sim\left(10^{8} \mathrm{GeV}\right)^{2}$ results in a similar fine-tuning penalty as tuning a single scalar mass such that $M_{Z} \sim 100 \mathrm{GeV}$. This, in essence, explains why the evidence for the SM and relaxion models are similar, if one considers only weak + conditions. Note that lowering the quadratic corrections by supersymmetrizing the SM and relaxion models see e.g. ref. [94] could favour relaxion models, as from eq. (5.1) a Bayes-factor might scale as the cut-off squared. Lowering the Planck mass, on the other hand, might help slightly less, as it would lower the bounds on scalar masses from eq. (4.14).

To further investigate this issue, we relaxed the Planck-scale cut-off, plotting evidence as a function of the cut-off in the SM and our QCD relaxion model in figure 2. By doing so, we wish to confirm that our QCD relaxion model would be favoured versus the SM, were the cut-off much lower than the Planck scale. We find in figure 2 that, although we previously found that the relaxion model was not favoured versus the SM with a Planckscale cut-off, if the cut-off were lowered in each model to about $10^{8} \mathrm{GeV}$, the relaxion 


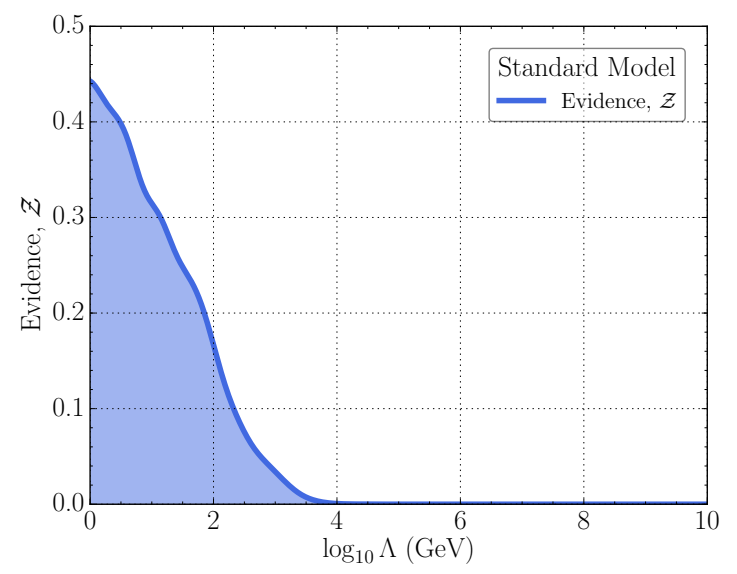

(a) SM.

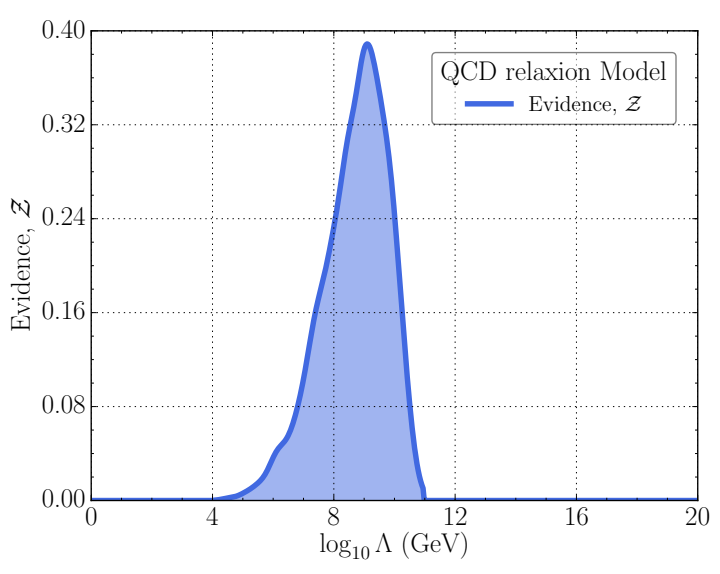

(b) Relaxion.

Figure 2. The evidence as a function of the cut-off, $\Lambda$, in (a) the SM and (b) a relaxion model. The evidence includes weak-scale and conditions. This illustrates that a relaxion model could be significantly favoured if the cut-off were lowered from the Planck scale to about $10^{8} \mathrm{GeV}$ e.g., by supersymmetrizing the SM and relaxion model. The evidences are plotted in arbitrary units.

model could be significantly favoured. In other words, the relaxion mechanism may solve the little-hierarchy problem in a supersymmetric model with soft-breaking masses at about $10^{8} \mathrm{GeV}$, but not the hierarchy problem by itself. Hence, in isolation, our QCD relaxion model cannot improve fine-tuning compared to the SM.

With a cut-off allowed to be as low as $10 \mathrm{TeV}$, considering weak-scale, conditions and decay, the Bayes-factor favours our QCD relaxion model by $10^{6}$ versus the SM and about $10^{31}$ versus the SM with Planck-scale quadratic corrections. Including low-scale inflation in e.g., a supersymmetrized relaxion model, however, might necessitate an inflaton mass $m_{\sigma} \ll M_{\mathrm{SUSY}}$. This little-hierarchy problem could scotch the Bayes-factor of $10^{6}$ in favour of the supersymmetrized relaxion model.

\subsection{Observables}

To illustrate the resolution of the hierarchy problem, in figure 3 we plot the priors for the $Z$-boson mass in the SM and our QCD relaxion model that result from the non-informative priors for Lagrangian parameters in table 2, that is,

$$
p\left(\log M_{Z} \mid M\right)=\int \delta\left(\log M_{Z}-\log M_{Z}(\boldsymbol{p})\right) p(\boldsymbol{p} \mid M) \prod \mathrm{d} \boldsymbol{p} .
$$

This illustrates their generic predictions for the weak scale. This would be numerically equivalent to the Bayesian evidence if our data were $\log M_{Z}$ and we approximated our measurement with a Dirac function. Whereas the SM favours a weak scale close to the Planck scale, the relaxion model results in considerable probability mass at scales much less than the Planck scale, resolving the hierarchy problem. We find that if the relaxion is the QCD axion, the posterior probability that $\left|\theta_{\mathrm{QCD}}\right| \lesssim 10^{-10}$ is negligible, confirming our expectations. 


\begin{tabular}{|cccccc|}
\hline Data-set & weak-scale & +=conditions & +=decay & +=theta & +=inflation \\
\hline $\mathcal{Z}(\mathrm{SM}+\sigma) \cdot \mathrm{GeV}$ & $10^{-34}$ & & & $10^{-45}$ & $10^{-53}$ \\
$\mathcal{Z}($ relaxion $) \cdot \mathrm{GeV}$ & $10^{-4}$ & $10^{-32}$ & & $10^{-42}$ & $10^{-77}$ \\
$\mathcal{Z}(\mathrm{QCD}$ relaxion $) \cdot \mathrm{GeV}$ & $10^{-4}$ & $10^{-34}$ & $10^{-39}$ & $\lll 10^{-39}$ & $\lll 10^{-81}$ \\
\hline$B($ relaxion $/ \mathrm{SM}+\sigma)$ & $10^{30}$ & $10^{2}$ & & $10^{2}$ & $10^{-25}$ \\
$B(\mathrm{QCD}$ relaxion $/ \mathrm{SM}+\sigma)$ & $10^{30}$ & 1 & $10^{-5}$ & $\lll 10^{5}$ & $\lll 10^{-28}$ \\
$B(\mathrm{QCD}$ relaxion/relaxion $)$ & 1 & $10^{-2}$ & $10^{-7}$ & $\lll 10^{3}$ & $\lll 10^{-3}$ \\
\hline$P($ relaxion/SM $+\sigma)$ & & $10^{-28}$ & & 1 & $10^{-27}$ \\
$P(\mathrm{QCD}$ relaxion/SM $+\sigma)$ & & $10^{-30}$ & $10^{-6}$ & $\lll 1$ & $10^{-33}$ \\
$P(\mathrm{QCD}$ relaxion/relaxion $)$ & & $10^{-2}$ & $10^{-6}$ & $\lll 1$ & $10^{-6}$ \\
\hline
\end{tabular}

Table 3. Evidences, $\mathcal{Z}$, Bayes-factors, $B$ and partial Bayes-factors, $P$, for the SM augmented with scalar-field inflation $(\mathrm{SM}+\sigma)$, a relaxion toy-model and a QCD relaxion toy-model. We apply data incrementally in five data-sets: $(i)$ the $Z$-boson mass (weak-scale), (ii) physicality conditions in relaxion models (conditions), (iii) constraints on the axion decay constant (decay), (iv) $\left|\theta_{\mathrm{QCD}}\right|$ (theta) and $(v)$ BICEP/Planck measurements of inflationary observables (inflation). A Bayesfactor is a ratio of evidences, indicating the change in relative plausibility of two models in light of all data considered thus far. A partial Bayes-factor is a ratio of Bayes-factors, indicating the change in relative plausibility of two models in light of incrementing the data by a single data-set. A ratio of greater than one indicates that a relaxion toy-model is favoured. We highlight our most important findings in blue: that relaxion toy-models are favoured by about $10^{30}$ by the $Z$-boson mass, but that once all constaints are included, that preference is reversed to about $10^{-25}$ against relaxion toy-models.

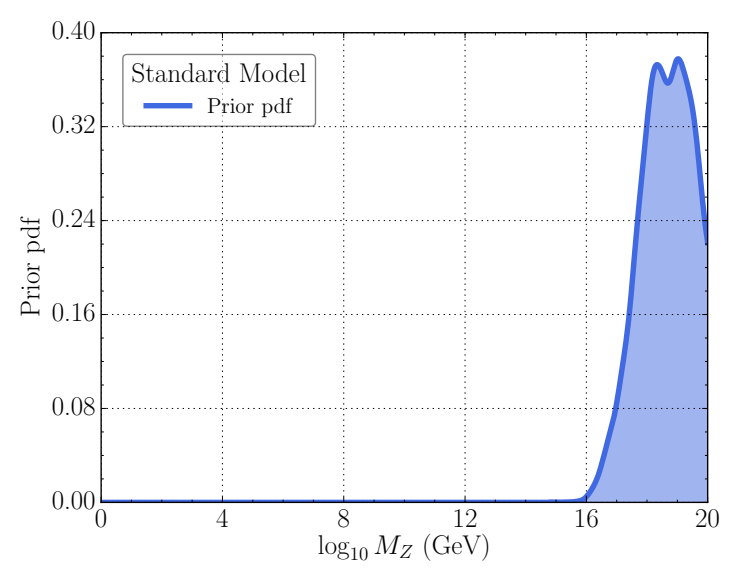

(a) SM.

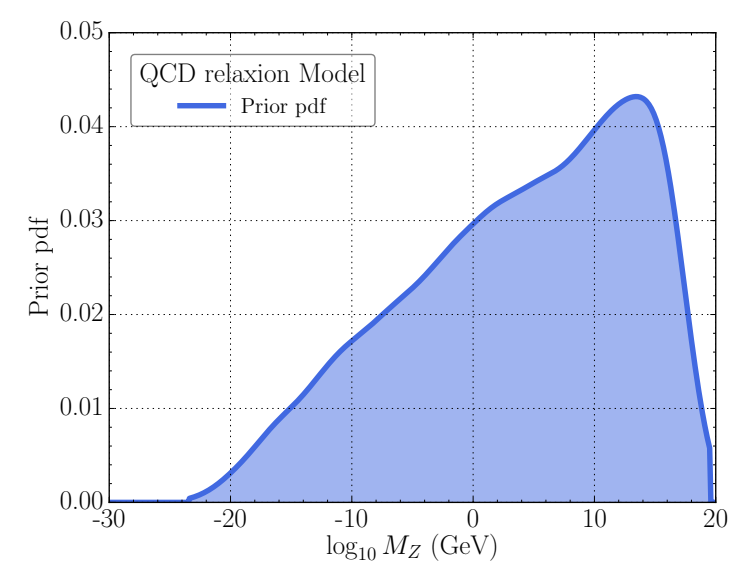

(b) Relaxion.

Figure 3. Prior distribution of $\log _{10}$ of the $Z$-boson mass in (a) the SM and (b) a QCD relaxion model including no data. The density at the correct weak scale in the relaxion model is much greater than that in the SM. This illustrates that the relaxion mechanism improves fine-tuning of the weak scale with respect to the SM. The densities are plotted in arbitrary units. 


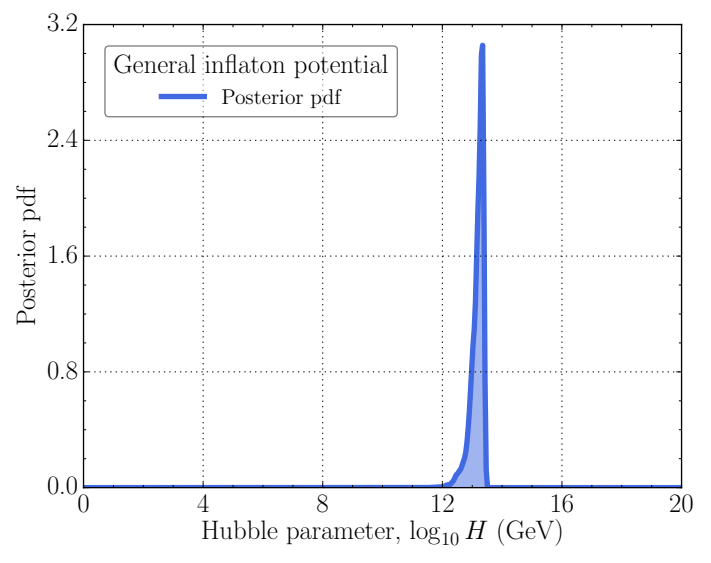

(a) Without $H<m_{b}$.

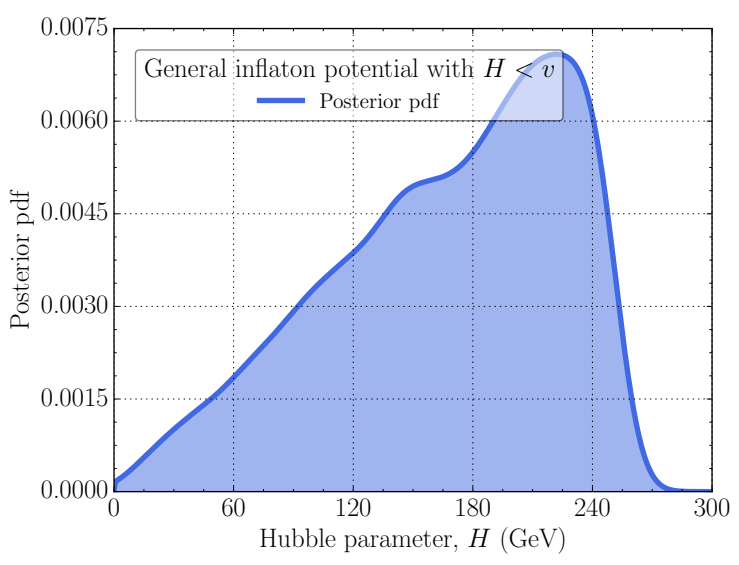

(b) With $H<m_{b}$.

Figure 4. Posterior distribution for the Hubble parameter, $H$, for a general renormalisable inflationary potential (eq. (4.10)). In (a), the constraint that the Hubble parameter is less than the height of the periodic barriers, $H<m_{b}$ (eq. (4.17)), is not applied; whereas in (b), it is applied. Both plots include Planck/BICEP measurements of $r, n_{s}$ and $A_{s}$.

To illustrate the fine-tuning required in the second epoch of inflation in a relaxion scenario, in figure 4 we plot the posterior distribution of the Hubble parameter $H$ with and without constraint that the Hubble parameter is less than the height of the periodic barriers, $H<m_{b}$ (eq. (4.17)), but with Planck/BICEP measurements of $r, n_{s}$ and $A_{s}$. In the unconstrained case in figure $4 \mathrm{a}$, the posterior shows preference for $H \ggg m_{b}$, as expected, resulting in a tiny evidence once $H<m_{b}$ is applied. Consequently, the Bayesfactor significantly favours inflation in the $\mathrm{SM}+\sigma$ model.

This can be understood analytically. As discussed in section 4.2 , for $H \lll M_{\mathrm{P}}$, we must have that $V(\langle\sigma\rangle) \lll M_{\mathrm{P}}^{4}$, where the potential is an order-four polynomial. The mass-squared term is typically Planckian as it received a quadratic correction. The general solution involves roots of a cubic equation. For simplicity, instead consider that $m_{3}^{3}=$ $m_{1}=0$. By dimensional analysis, the result must be that $V(\langle\sigma\rangle)=f(\lambda) m_{2}^{4} \sim M_{P}^{4}$. In fact, the result is that $V(\langle\sigma\rangle)=m_{2}^{4} /\left(4 \lambda_{\sigma}\right)$. Other than by extreme fine-tuning of the mass-squared (which might not agree with Planck/BICEP measurements of $r, n_{s}$ and $A_{s}$ ), there is no chance that $H \lll m_{b}$. On the other hand, consider that all dimensional parameters are equal to the mass-squared term, $\left|m_{1}\right|=\left|m_{2}\right|=\left|m_{3}\right|$. Again, it must be that $V(\langle\sigma\rangle)=f(\lambda) m_{2}^{4} \sim M_{P}^{4}$, though in this case the function $f$ is rather complicated. In any case, one must still require fine-tuning such that $V(\langle\sigma\rangle) \sim M_{\mathrm{P}}^{4}$ is avoided.

\section{Discussion and conclusions}

We constructed models that utilised a relaxation mechanism recently proposed by Graham et al. to solve the hierarchy problem. Unfortunately, finding the weak scale in relaxion models involves solving a transcendental equation with numerical methods. We presented 
an analytic expression for an interval bounding the weak scale and an analytic expression for a lower bound on $\left|\theta_{\mathrm{QCD}}\right|$, confirming that $\left|\theta_{\mathrm{QCD}}\right| \approx \pi / 2$ if the relaxion is the QCD axion.

We performed the first statistical analysis of a relaxion model by scanning relaxion models' parameter spaces with the nested sampling algorithm, considering data from measurements of the weak scale, the axion decay constant, $\left|\theta_{\mathrm{QCD}}\right|$ and BICEP/Planck measurements of inflationary observables $r, n_{s}$ and $A_{s}$. This resulted in so-called Bayesian evidences for our relaxion models augmented with scalar-field inflation. In a similar manner, we calculated Bayesian evidences for the SM augmented with scalar-field inflation.

We found that the Bayes-factors - ratios of Bayesian evidences that indicate how one ought to update one's relative prior belief in two models - favoured relaxion models versus the SM by a colossal factor of about $10^{30}$ if one considered only the weak scale. Once we included physicality conditions upon inflation during relaxation, however, the Bayesfactors were decimated to about 100 for the non-QCD relaxion model and about 1 for the QCD relaxion model. The physicality condition on the energy density during the relaxion mechanism results, in fact, in a fine-tuning penalty similar to that for the hierarchy problem in the SM, as demonstrated in eq. (5.1). The resulting Bayes-factors are then of order unity.

Constraints upon the QCD relaxion decay constant and $\left|\theta_{\mathrm{QCD}}\right|$ severely impair the plausibility of the QCD relaxion model, whereas inflationary observables measured by BICEP/Planck demolish that of the surviving, non-QCD relaxion model. In this regard, we find that the SM augmented with scalar-field inflation is favoured by a Bayes-factor of about $10^{25}$ with respect to the non-QCD relaxion model. This stems from a constraint upon the Hubble parameter during inflation: within the relaxion framework the Hubble parameter must be fine-tuned to $H \lll M_{\mathrm{P}}$, in order to prevent inflation from destroying the periodic barriers in the relaxion potential. In contrast, the polynomial inflationary potentials supported by current observations generically predict that $H \lesssim M_{\mathrm{P}}$, as explained in figure 4 and the accompanying discussion.

Thus, whilst the analysed relaxion models indeed solve the hierarchy problem leading to Bayes-factors of about $10^{30}$ in their favour, the same Bayes-factors are scotched by constraints upon parameters in the relaxion potential and the Hubble parameter during inflation, ultimately leading to a Bayes-factor of about $10^{25}$ in favour of the SM augmented with scalar-field inflation. We anticipate, furthermore, that detailed consideration of baryogenesis and thermal effects (including the disastrous possibility of reheating restoring electroweak symmetry) would further damage the plausibility of relaxion models and conclude that the required unconventional cosmology is the Achilles' heel of the relaxion mechanism.

To conclude, we also remark that our results stand in the absence of a clear UVcompletion for the considered relaxion models and that the lack of information regarding the origins of the parameters in the relaxion Lagrangian was encoded in the adopted uninformative priors. If such a UV-completion were available, it would possibly dictate a different choice of priors that could re-establish the plausibility of the relaxion framework. 


\section{Acknowledgments}

We thank Kristjan Kannike for helpful discussions. LM and MR are supported by the grants IUT23-6, PUTJD110 and by EU through the ERDF CoE program. AF, GW and CB are in part supported by the ARC Centre of Excellence for Particle Physics at the Tera-scale.

Open Access. This article is distributed under the terms of the Creative Commons Attribution License (CC-BY 4.0), which permits any use, distribution and reproduction in any medium, provided the original author(s) and source are credited.

\section{References}

[1] P.W. Graham, D.E. Kaplan and S. Rajendran, Cosmological relaxation of the electroweak scale, Phys. Rev. Lett. 115 (2015) 221801 [arXiv:1504.07551] [INSPIRE].

[2] L.F. Abbott, A mechanism for reducing the value of the cosmological constant, Phys. Lett. B 150 (1985) 427 [INSPIRE].

[3] G. Dvali and A. Vilenkin, Cosmic attractors and gauge hierarchy, Phys. Rev. D 70 (2004) 063501 [hep-th/0304043] [INSPIRE].

[4] G. Dvali, Large hierarchies from attractor vacua, Phys. Rev. D 74 (2006) 025018 [hep-th/0410286] [INSPIRE].

[5] S. Weinberg, Implications of dynamical symmetry breaking, Phys. Rev. D 13 (1976) 974 [INSPIRE].

[6] S. Weinberg, Implications of dynamical symmetry breaking: an addendum, Phys. Rev. D 19 (1979) 1277 [INSPIRE].

[7] L. Susskind, Dynamics of spontaneous symmetry breaking in the Weinberg-Salam theory, Phys. Rev. D 20 (1979) 2619 [inSPIRE].

[8] E. Gildener, Gauge symmetry hierarchies, Phys. Rev. D 14 (1976) 1667 [InSPIRE].

[9] E. Witten, Dynamical breaking of supersymmetry, Nucl. Phys. B 188 (1981) 513 [INSPIRE].

[10] V. Mukhanov, Physical foundations of cosmology, Cambridge University Press, Oxford U.K. (2005).

[11] S. Di Chiara, K. Kannike, L. Marzola, A. Racioppi, M. Raidal and C. Spethmann, Relaxion cosmology and the price of fine-tuning, Phys. Rev. D 93 (2016) 103527 [arXiv:1511.02858] [INSPIRE].

[12] J. Jaeckel, V.M. Mehta and L.T. Witkowski, Musings on cosmological relaxation and the hierarchy problem, Phys. Rev. D 93 (2016) 063522 [arXiv:1508.03321] [INSPIRE].

[13] R. Barbieri and G.F. Giudice, Upper bounds on supersymmetric particle masses, Nucl. Phys. B 306 (1988) 63 [inSPIRE].

[14] J.R. Ellis, K. Enqvist, D.V. Nanopoulos and F. Zwirner, Observables in low-energy superstring models, Mod. Phys. Lett. A 1 (1986) 57 [inSPIRE].

[15] B.C. Allanach, K. Cranmer, C.G. Lester and A.M. Weber, Natural priors, CMSSM fits and LHC weather forecasts, JHEP 08 (2007) 023 [arXiv:0705.0487] [INSPIRE].

[16] M.E. Cabrera, J.A. Casas and R. Ruiz de Austri, Bayesian approach and naturalness in MSSM analyses for the LHC, JHEP 03 (2009) 075 [arXiv:0812.0536] [INSPIRE]. 
[17] A. Fowlie, CMSSM, naturalness and the "fine-tuning price" of the Very Large Hadron Collider, Phys. Rev. D 90 (2014) 015010 [arXiv: 1403.3407] [INSPIRE].

[18] S. Fichet, Quantified naturalness from Bayesian statistics, Phys. Rev. D 86 (2012) 125029 [arXiv:1204.4940] [INSPIRE].

[19] M.E. Cabrera, Bayesian study and naturalness in MSSM forecast for the LHC, in Proceedings, $45^{\text {th }}$ Rencontres de Moriond on Electroweak Interactions and Unified Theories, (2010) [arXiv: 1005.2525] [INSPIRE].

[20] A. Fowlie, The little-hierarchy problem is a little problem: understanding the difference between the big- and little-hierarchy problems with Bayesian probability, arXiv:1506.03786 [INSPIRE].

[21] D. Kim, P. Athron, C. Balázs, B. Farmer and E. Hutchison, Bayesian naturalness of the CMSSM and CNMSSM, Phys. Rev. D 90 (2014) 055008 [arXiv:1312.4150] [INSPIRE].

[22] A. Fowlie, Is the CNMSSM more credible than the CMSSM?, Eur. Phys. J. C 74 (2014) 3105 [arXiv: 1407.7534] [INSPIRE].

[23] J. Earman, Bayes or bust?: a critical examination of Bayesian confirmation theory, MIT Press, U.S.A. (1992).

[24] E.T. Jaynes, Probability theory: the logic of science, Cambridge University Press, Cambridge U.K. (2003).

[25] P. Gregory, Bayesian logical data analysis for the physical sciences, Cambridge University Press, Cambridge U.K. (2005).

[26] D.E. Lopez-Fogliani, L. Roszkowski, R. Ruiz de Austri and T.A. Varley, A Bayesian analysis of the constrained NMSSM, Phys. Rev. D 80 (2009) 095013 [arXiv:0906.4911] [INSPIRE].

[27] K. Kowalska, S. Munir, L. Roszkowski, E.M. Sessolo, S. Trojanowski and Y.-L.S. Tsai, Constrained next-to-minimal supersymmetric Standard Model with a $126 \mathrm{GeV}$ Higgs boson: a global analysis, Phys. Rev. D 87 (2013) 115010 [arXiv:1211.1693] [INSPIRE].

[28] A.J. Williams, Explaining the Fermi galactic centre excess in the CMSSM, arXiv: 1510.00714 [INSPIRE].

[29] R. Diamanti, M.E.C. Catalan and S. Ando, Dark matter protohalos in a nine parameter MSSM and implications for direct and indirect detection, Phys. Rev. D 92 (2015) 065029 [arXiv: 1506. 01529] [INSPIRE].

[30] M.E. Cabrera-Catalan, S. Ando, C. Weniger and F. Zandanel, Indirect and direct detection prospect for TeV dark matter in the nine parameter MSSM, Phys. Rev. D 92 (2015) 035018 [arXiv: 1503.00599] [INSPIRE].

[31] J.A. Casas, J.M. Moreno, S. Robles, K. Rolbiecki and B. Zaldívar, What is a natural SUSY scenario?, JHEP 06 (2015) 070 [arXiv: 1407.6966] [INSPIRE].

[32] L. Roszkowski, E.M. Sessolo and A.J. Williams, What next for the CMSSM and the NUHM: improved prospects for superpartner and dark matter detection, JHEP 08 (2014) 067 [arXiv: 1405.4289] [INSPIRE].

[33] C. Strege et al., Profile likelihood maps of a 15-dimensional MSSM, JHEP 09 (2014) 081 [arXiv: 1405.0622] [INSPIRE]. 
[34] A. Fowlie and M. Raidal, Prospects for constrained supersymmetry at $\sqrt{s}=33 \mathrm{TeV}$ and $\sqrt{s}=100$ TeV proton-proton super-colliders, Eur. Phys. J. C 74 (2014) 2948 [arXiv: 1402.5419] [INSPIRE].

[35] S.S. AbdusSalam, Stop-mass prediction in naturalness scenarios within MSSM-25, Int. J. Mod. Phys. A 29 (2014) 1450160 [arXiv:1312.7830] [InSPIRE].

[36] M.E. Cabrera, A. Casas, R. Ruiz de Austri and G. Bertone, LHC and dark matter phenomenology of the NUGHM, JHEP 12 (2014) 114 [arXiv:1311.7152] [INSPIRE].

[37] C. Arina and M.E. Cabrera, Multi-lepton signatures at LHC from sneutrino dark matter, JHEP 04 (2014) 100 [arXiv:1311.6549] [INSPIRE].

[38] R. Ruiz de Austri and C. Pérez de los Heros, Impact of nucleon matrix element uncertainties on the interpretation of direct and indirect dark matter search results, JCAP 11 (2013) 049 [arXiv: 1307.6668] [INSPIRE].

[39] A. Fowlie, K. Kowalska, L. Roszkowski, E.M. Sessolo and Y.-L.S. Tsai, Dark matter and collider signatures of the MSSM, Phys. Rev. D 88 (2013) 055012 [arXiv:1306.1567] [INSPIRE].

[40] M.E. Cabrera, J.A. Casas and R. Ruiz de Austri, The health of SUSY after the Higgs discovery and the XENON100 data, JHEP 07 (2013) 182 [arXiv:1212.4821] [INSPIRE].

[41] C. Strege, G. Bertone, F. Feroz, M. Fornasa, R. Ruiz de Austri and R. Trotta, Global fits of the CMSSM and NUHM including the LHC Higgs discovery and new XENON100 constraints, JCAP 04 (2013) 013 [arXiv: 1212.2636] [INSPIRE].

[42] C. Balázs and S.K. Gupta, Peccei-Quinn violating minimal supergravity and a $126 \mathrm{GeV}$ Higgs boson, Phys. Rev. D 87 (2013) 035023 [arXiv:1212.1708] [INSPIRE].

[43] A. Fowlie et al., The CMSSM favoring new territories: the impact of new LHC limits and a 125 GeV Higgs, Phys. Rev. D 86 (2012) 075010 [arXiv: 1206. 0264] [INSPIRE].

[44] C. Balázs, A. Buckley, D. Carter, B. Farmer and M. White, Should we still believe in constrained supersymmetry?, Eur. Phys. J. C 73 (2013) 2563 [arXiv:1205.1568] [INSPIRE].

[45] L. Roszkowski, E.M. Sessolo and Y.-L.S. Tsai, Bayesian implications of current LHC supersymmetry and dark matter detection searches for the constrained MSSM, Phys. Rev. D 86 (2012) 095005 [arXiv: 1202.1503] [INSPIRE].

[46] C. Strege, G. Bertone, D.G. Cerdeno, M. Fornasa, R. Ruiz de Austri and R. Trotta, Updated global fits of the CMSSM including the latest LHC SUSY and Higgs searches and XENON100 data, JCAP 03 (2012) 030 [arXiv:1112.4192] [INSPIRE].

[47] A. Fowlie, A. Kalinowski, M. Kazana, L. Roszkowski and Y.L.S. Tsai, Bayesian implications of current LHC and XENON100 search limits for the constrained MSSM, Phys. Rev. D 85 (2012) 075012 [arXiv:1111.6098] [INSPIRE].

[48] G. Bertone, D.G. Cerdeno, M. Fornasa, L. Pieri, R. Ruiz de Austri and R. Trotta, Complementarity of indirect and accelerator dark matter searches, Phys. Rev. D 85 (2012) 055014 [arXiv: 1111.2607] [INSPIRE].

[49] M.E. Cabrera, J.A. Casas, V.A. Mitsou, R. Ruiz de Austri and J. Terron, Histogram comparison as a powerful tool for the search of new physics at LHC. Application to CMSSM, JHEP 04 (2012) 133 [arXiv:1109.3759] [INSPIRE]. 
[50] B.C. Allanach and M.J. Dolan, Supersymmetry with prejudice: fitting the wrong model to LHC data, Phys. Rev. D 86 (2012) 055022 [arXiv:1107.2856] [InSPIRE].

[51] G. Bertone, D.G. Cerdeno, M. Fornasa, R. Ruiz de Austri, C. Strege and R. Trotta, Global fits of the CMSSM including the first LHC and XENON100 data, JCAP 01 (2012) 015 [arXiv:1107.1715] [INSPIRE].

[52] D.T. Cumberbatch, D.E. Lopez-Fogliani, L. Roszkowski, R. Ruiz de Austri and Y.-L.S. Tsai, Is light neutralino as dark matter still viable?, arXiv:1107.1604 [INSPIRE].

[53] A. Fowlie and L. Roszkowski, Reconstructing ATLAS SU3 in the CMSSM and relaxed phenomenological supersymmetry models, arXiv:1106.5117 [INSPIRE].

[54] B.C. Allanach, Impact of CMS multi-jets and missing energy search on CMSSM fits, Phys. Rev. D 83 (2011) 095019 [arXiv: 1102.3149] [InSPIRE].

[55] F. Feroz, K. Cranmer, M. Hobson, R. Ruiz de Austri and R. Trotta, Challenges of profile likelihood evaluation in multi-dimensional SUSY scans, JHEP 06 (2011) 042 [arXiv: 1101.3296] [INSPIRE].

[56] J. Ripken, J. Conrad and P. Scott, Implications for constrained supersymmetry of combined H.E.S.S. observations of dwarf galaxies, the galactic halo and the galactic centre, JCAP 11 (2011) 004 [arXiv: 1012.3939] [INSPIRE].

[57] M.E. Cabrera, J.A. Casas, R. Ruiz de Austri and R. Trotta, Quantifying the tension between the Higgs mass and $(g-2)_{\mu}$ in the CMSSM, Phys. Rev. D 84 (2011) 015006 [arXiv: 1011.5935] [INSPIRE].

[58] Y. Akrami, C. Savage, P. Scott, J. Conrad and J. Edsjo, Statistical coverage for supersymmetric parameter estimation: a case study with direct detection of dark matter, JCAP 07 (2011) 002 [arXiv: 1011.4297] [INSPIRE].

[59] M.E. Cabrera, J.A. Casas and R. Ruiz d Austri, MSSM forecast for the LHC, JHEP 05 (2010) 043 [arXiv: 0911.4686] [inSPIRE].

[60] Y. Akrami, P. Scott, J. Edsjo, J. Conrad and L. Bergstrom, A profile likelihood analysis of the constrained MSSM with genetic algorithms, JHEP 04 (2010) 057 [arXiv:0910.3950] [INSPIRE].

[61] L. Roszkowski, R. Ruiz de Austri and R. Trotta, Efficient reconstruction of CMSSM parameters from LHC data: a case study, Phys. Rev. D 82 (2010) 055003 [arXiv:0907.0594] [INSPIRE].

[62] L. Roszkowski, R. Ruiz de Austri, R. Trotta, Y.-L.S. Tsai and T.A. Varley, Global fits of the non-universal Higgs model, Phys. Rev. D 83 (2011) 015014 [Erratum ibid. D 83 (2011) 039901] [arXiv: 0903.1279] [INSPIRE].

[63] R. Trotta, F. Feroz, M.P. Hobson, L. Roszkowski and R. Ruiz de Austri, The impact of priors and observables on parameter inferences in the constrained MSSM, JHEP 12 (2008) 024 [arXiv: 0809.3792] [INSPIRE].

[64] F. Feroz, B.C. Allanach, M. Hobson, S.S. AbdusSalam, R. Trotta and A.M. Weber, Bayesian selection of sign $\mu$ within MSUGRA in global fits including WMAP5 results, JHEP 10 (2008) 064 [arXiv:0807.4512] [INSPIRE].

[65] B.C. Allanach and D. Hooper, Panglossian prospects for detecting neutralino dark matter in light of natural priors, JHEP 10 (2008) 071 [arXiv:0806.1923] [INSPIRE]. 
[66] B.C. Allanach, M.J. Dolan and A.M. Weber, Global fits of the large volume string scenario to WMAP5 and other indirect constraints using Markov chain Monte Carlo, JHEP 08 (2008) 105 [arXiv:0806.1184] [inSPIRE].

[67] B.C. Allanach, SUSY predictions and SUSY tools at the LHC, Eur. Phys. J. C 59 (2009) 427 [arXiv: 0805. 2088] [INSPIRE].

[68] L. Roszkowski, R. Ruiz de Austri and R. Trotta, Implications for the constrained MSSM from a new prediction for $b \rightarrow s \gamma$, JHEP 07 (2007) 075 [arXiv:0705.2012] [INSPIRE].

[69] L. Roszkowski, R. Ruiz de Austri and R. Trotta, On the detectability of the CMSSM light Higgs boson at the Tevatron, JHEP 04 (2007) 084 [hep-ph/0611173] [INSPIRE].

[70] R. Trotta, Bayes in the sky: Bayesian inference and model selection in cosmology, Contemp. Phys. 49 (2008) 71 [arXiv: 0803.4089] [INSPIRE].

[71] J. Martin, C. Ringeval, R. Trotta and V. Vennin, The best inflationary models after Planck, JCAP 03 (2014) 039 [arXiv: 1312.3529] [INSPIRE].

[72] J. Martin, C. Ringeval, R. Trotta and V. Vennin, Compatibility of Planck and BICEP2 in the light of inflation, Phys. Rev. D 90 (2014) 063501 [arXiv:1405.7272] [inSPIRE].

[73] W. Jefferys and J. Berger, Sharpening Occam's razor on a Bayesian strop, Bull. Amer. Astron. Soc. 23 (1991) 1259.

[74] J.R. Espinosa, C. Grojean, G. Panico, A. Pomarol, O. Pujolàs and G. Servant, Cosmological Higgs-axion interplay for a naturally small electroweak scale, Phys. Rev. Lett. 115 (2015) 251803 [arXiv: 1506.09217] [INSPIRE].

[75] G. 't Hooft, Naturalness, chiral symmetry, and spontaneous chiral symmetry breaking, NATO Sci. Ser. B 59 (1980) 135 [INSPIRE].

[76] R.S. Gupta, Z. Komargodski, G. Perez and L. Ubaldi, Is the relaxion an axion?, JHEP 02 (2016) 166 [arXiv: 1509.00047] [INSPIRE].

[77] L.E. Ibáñez, M. Montero, A. Uranga and I. Valenzuela, Relaxion monodromy and the weak gravity conjecture, JHEP 04 (2016) 020 [arXiv: 1512.00025] [INSPIRE].

[78] R.D. Peccei and H.R. Quinn, CP conservation in the presence of instantons, Phys. Rev. Lett. 38 (1977) 1440 [INSPIRE].

[79] Particle Data Group collaboration, K.A. Olive et al., Review of particle physics, Chin. Phys. C 38 (2014) 090001 [INSPIRE].

[80] J.F. Donoghue, CP violation and the limits of the Standard Model: (TASI-94) Proceedings of the 1994 Theoretical Advanced Study Institute in Elementary Particle Physics, World Scientific, Singapore (1995).

[81] E. Hardy, Electroweak relaxation from finite temperature, JHEP 11 (2015) 077 [arXiv: 1507.07525] [INSPIRE].

[82] D.J. Gross, R.D. Pisarski and L.G. Yaffe, $Q C D$ and instantons at finite temperature, Rev. Mod. Phys. 53 (1981) 43 [INSPIRE].

[83] M. Quirós, Finite temperature field theory and phase transitions, in High energy physics and cosmology. Proceedings, Summer School, Trieste Italy June 29-July 17 1998, pg. 187 [hep-ph/9901312] [INSPIRE]. 
[84] A.D. Sakharov, Violation of CP invariance, $c$ asymmetry and baryon asymmetry of the universe, Pisma Zh. Eksp. Teor. Fiz. 5 (1967) 32 [JETP Lett. 5 (1967) 24] [Sov. Phys. Usp. 34 (1991) 392] [Usp. Fiz. Nauk 161 (1991) 61] [InSPIRE].

[85] A. Riotto, Theories of baryogenesis, in High energy physics and cosmology. Proceedings, Summer School, Trieste Italy June 29-July 17 1998, pg. 326 [hep-ph/9807454] [INSPIRE].

[86] R. Rangarajan and D.V. Nanopoulos, Inflationary baryogenesis, Phys. Rev. D 64 (2001) 063511 [hep-ph/0103348] [inSPIRE].

[87] S. H.-S. Alexander, M.E. Peskin and M.M. Sheikh-Jabbari, Leptogenesis from gravity waves in models of inflation, Phys. Rev. Lett. 96 (2006) 081301 [hep-th/0403069] [INSPIRE].

[88] H.H. Patel and M.J. Ramsey-Musolf, Baryon washout, electroweak phase transition and perturbation theory, JHEP 07 (2011) 029 [arXiv:1101.4665] [INSPIRE].

[89] S.P. Patil and P. Schwaller, Relaxing the electroweak scale: the role of broken dS symmetry, JHEP 02 (2016) 077 [arXiv: 1507.08649] [INSPIRE].

[90] O. Matsedonskyi, Mirror cosmological relaxation of the electroweak scale, JHEP 01 (2016) 063 [arXiv: 1509.03583] [INSPIRE].

[91] D.E. Kaplan and R. Rattazzi, Large field excursions and approximate discrete symmetries from a clockwork axion, Phys. Rev. D 93 (2016) 085007 [arXiv:1511.01827] [INSPIRE].

[92] K. Choi and S.H. Im, Realizing the relaxion from multiple axions and its UV completion with high scale supersymmetry, JHEP 01 (2016) 149 [arXiv:1511.00132] [INSPIRE].

[93] O. Antipin and M. Redi, The half-composite two Higgs doublet model and the relaxion, JHEP 12 (2015) 031 [arXiv: 1508.01112] [INSPIRE].

[94] B. Batell, G.F. Giudice and M. McCullough, Natural heavy supersymmetry, JHEP 12 (2015) 162 [arXiv: 1509.00834] [INSPIRE].

[95] N. Fonseca, L. de Lima, C.S. Machado and R.D. Matheus, Large field excursions from a few site relaxion model, Phys. Rev. D 94 (2016) 015010 [arXiv: 1601.07183] [INSPIRE].

[96] J.L. Evans, T. Gherghetta, N. Nagata and Z. Thomas, Naturalizing supersymmetry with a two-field relaxion mechanism, arXiv: 1602.04812 [INSPIRE].

[97] J. Martin, C. Ringeval and V. Vennin, Encyclopæedia inflationaris, Phys. Dark Univ. 5-6 (2014) 75 [arXiv: 1303.3787] [INSPIRE].

[98] A.R. Liddle and D.H. Lyth, COBE, gravitational waves, inflation and extended inflation, Phys. Lett. B 291 (1992) 391 [astro-ph/9208007] [INSPIRE].

[99] A.R. Liddle and D.H. Lyth, Cosmological inflation and large scale structure, Cambridge University Press, Cambridge U.K. (2000).

[100] Planck collaboration, P.A.R. Ade et al., Planck 2013 results. XXII. Constraints on inflation, Astron. Astrophys. 571 (2014) A22 [arXiv: 1303.5082] [INSPIRE].

[101] R. Bousso, TASI lectures on the cosmological constant, Gen. Rel. Grav. 40 (2008) 607 [arXiv:0708.4231] [INSPIRE].

[102] J. Buchner et al., X-ray spectral modelling of the AGN obscuring region in the CDFS: Bayesian model selection and catalogue, Astron. Astrophys. 564 (2014) A125 [arXiv: 1402.0004] [INSPIRE]. 
[103] F. Feroz, M.P. Hobson, E. Cameron and A.N. Pettitt, Importance nested sampling and the MultiNest algorithm, arXiv:1306.2144 [INSPIRE].

[104] F. Feroz and M.P. Hobson, Multimodal nested sampling: an efficient and robust alternative to MCMC methods for astronomical data analysis, Mon. Not. Roy. Astron. Soc. 384 (2008) 449 [arXiv: 0704.3704] [INSPIRE].

[105] F. Feroz, M.P. Hobson and M. Bridges, MultiNest: an efficient and robust Bayesian inference tool for cosmology and particle physics, Mon. Not. Roy. Astron. Soc. 398 (2009) 1601 [arXiv: 0809.3437] [INSPIRE].

[106] J. Skilling, Nested sampling, AIP Conf. Proc. 735 (2004) 395.

[107] J. Skilling, Nested sampling for general Bayesian computation, Bayesian Anal. 1 (2006) 833.

[108] M. Dine, Supersymmetry and string theory: beyond the Standard Model, Cambridge University Press, Cambridge U.K. (2007).

[109] BICEP2 and PlAnCK collaborations, P.A.R. Ade et al., Joint analysis of BICEP2/Keck Array and Planck data, Phys. Rev. Lett. 114 (2015) 101301 [arXiv:1502.00612] [InSPIRE].

[110] Planck collaboration, P.A.R. Ade et al., Planck 2015 results. XIII. Cosmological parameters, arXiv:1502.01589 [INSPIRE]. 